\title{
Potencial bioativo dos flavonoides vitexina, tilirosideo e 5,7-dihidroxi-3,8,4'-trimetoxi no tratamento de doenças crônicas e neoplasias do sistema gastrointestinal e excretor
}

Properties of the flavonoids vitexin, tiliroside and 5,7-dihydroxy-3,8,4' trimethoxy in the treatment of chronic diseases and neoplasia of the gastrointestinal and excretory systems

Propiedades de los flavonoides vitexina, tilirosideo y 5,7-dihidroxi-3,8,4 trimetoxi en el tratamiento de enfermedades crónicas y neoplasias de los sistemas gastrointestinal y excretorio

Aleson Pereira de SOUSA ${ }^{1}$

Diégina Araújo FERNANDES ${ }^{2}$

Maria Denise Leite FERREIRA ${ }^{2}$

Laísa Vilar CORDEIRO ${ }^{2}$

Helivaldo Diógenes da Silva SOUZA ${ }^{2}$

Maria de Fátima Vanderlei de SOUZA²

Hilzeth de Luna Freire PESSOA ${ }^{2}$

Abrahão Alves de OLIVEIRA FILHO

Rita de Cássia da Silveira e SÁ 1

'Programa de Pós-Graduação em Desenvolvimento e Inovação Tecnológica em Medicamentos, Universidade Federal da Paraíba (DITM/UFPB) 58051-970 - João Pessoa - PB, Brasil

${ }^{2}$ Centro de Ciências da Saúde, Universidade Federal da Paraíba, 58033-455, João Pessoa-PB, Brasil

${ }^{3}$ Professor Doutor, Unidade Acadêmica de Ciências Biológicas, Centro de Saúde e Tecnologia Rural, Universidade Federal de Campina Grande - UFCG 58428-830 Campina Grande - PB, Brasil

\section{Resumo}

As doenças crônicas representam o principal grupo de doenças com elevados índices de mortalidade. Dessa forma, a utilização de plantas medicinais por adultos no tratamento de enfermidades ou amenização de seus sintomas é uma prática alternativa que vem sendo adotada mundialmente. $\mathrm{O}$ avanço científico e a modernização contribuem substancialmente para descoberta de substâncias naturais para produção de novos fármacos, com uma grande possibilidade de terem menor grau de toxicidade. Algumas dessas plantas são ricas em moléculas dotadas de potencial biotecnológico, como os flavonoides. O presente estudo avaliou o perfil bioativo in silico de três flavonoides: tilirosídeo (Kaempferol-3-O- $\beta$-D-(6"-Ep-coumaril) glucopiranosideo), Pg-1 (5,7-dihidroxi-3,8,4'-trimetoxi) e vitexina (5,7,4'-trihidroxiflavona-8-C- $\beta$ glucopiranosideo). Foi abordada a ação preditiva antineoplásica em órgãos do sistema gastrointestinal e urinário e a atividade protetora contra doenças crônicas (diabetes, hipertensão, catarata, osteoporose, hipercolesterolemia, disfunção de tireoide e doenças renais). $O$ estudo demonstrou que a vitexina apresentou efeito antidiabetes (tipo II) e antihipercolesterolemia; o tilirosideo, atividade antidiabetes (tipo I), antihipercolesterolemia, antihipóxico, antinefrítico e antiosteoporótico, e $\circ \mathrm{Pg}-1$, ação anticatarata, anti-hipertensiva e antitireoidiana. A avaliação da probabilidade do potencial antineoplásico mostrou que a vitexina possui melhor potencial anticâncer no pâncreas, estômago, bexiga e rim, e efeito anti - Helicobacter pylori. O Pg-1 apresentou maior atividade anticâncer no cólon e na região cólon-retal. O tilirosideo, apesar de exibir menor efeito em relação às demais substâncias, evidenciou atividade satisfatória anticâncer pancreática e cólon-retal. Portanto, estas moléculas podem tornar-se importantes candidatas a fármacos naturais como recurso terapêutico de combate a doenças crônicas e ao câncer do sistema gastrointestinal e urinário.

Descritores: Produtos Naturais; Simulação por Computador; Doença Crônica.

\section{Abstract}

The chronic diseases represent the main group of diseases with high mortality rates. Thus, the use of medicinal plants to treat different ailments or to ameliorate their symptoms by the adult population is an alternative practice that is being used worldwide. Scientific advances and modernization contribute substantially to the discovery of natural substances for the production of new drugs with a possible low toxicity risk. Some of these plants are rich in molecules with biotechnological potential, such as flavonoids. The present study evaluated the in silico bioactive profile of three flavonoids: tiliroside (Kaempferol-3-O- $\beta$-D-(6"-Ep-coumaryl) glucopyranoside), Pg-1 (5,7-dihydroxy-3,8,4'-trimethoxy) and vitexin (5,7,4'-trihydroxyflavone-8-C- $\beta$-glucopyranoside). The antineoplastic predictive action on organs of the gastrointestinal and urinary systems and the protective activity against chronic diseases (diabetes, hypertension, cataracts, osteoporosis, hypercholesterolemia, thyroid dysfunction and kidney diseases) were addressed. The study demonstrated that vitexin had anti-diabetes (type II) and antihypercholesterolemia action; tiliroside exhibited antidiabetes (type I), antihypercholesterolemia, antihypoxic, antinephritic and antiosteoporotic activity, and. Pg-1 showed anti-cataract, anti-hypertensive and anti-thyroid effect. The evaluation of the probability of the antineoplastic potential demonstrated that vitexin has a better anticancer potential in the pancreas, stomach, bladder and kidney in addition to anti-Helicobacter pylori effect. Pg-1 showed higher activity against colon and colonrectal cancer. Tiliroside, despite exhibiting lower effect than the other substances, displayed satisfactory pancreatic and colonrectal anticancer activity. Therefore, these molecules are possible important new drug candidates from natural products as a therapeutic resource against chronic diseases and the gastrointestinal and urinary systems cancers.

\section{Descriptors: Biological Products; Computer Simulation; Chronic Disease.}

\section{Resumen}

Las enfermedades crónicas representan el principal grupo de enfermedades con altas tasas de mortalidad. Así, el uso de plantas medicinales por parte de adultos en el tratamiento de enfermedades o el alivio de sus síntomas es una práctica alternativa que se ha adoptado a nivel mundial. Los avances científicos y la modernización contribuyen sustancialmente al descubrimiento de sustancias naturales para la producción de nuevos fármacos, con una gran posibilidad de tener un menor grado de toxicidad. Algunas de estas plantas son ricas en moléculas con potencial biotecnológico, como los flavonoides. El presente estudio evaluó el perfil bioactivo in silico de tres flavonoides: tilirosida (Kaempferol3-O-ß-D- (6 "-Ep-cumaril) glucopiranósido), Pg-1 (5,7-dihidroxi-3,8, 4'-trimetoxi) y vitexina (5,7,4'-trihidroxiflavona-8-C- $\beta$-glucopiranósido). Se abordó la acción predictiva antineoplásica sobre órganos del aparato gastrointestinal y urinario y la actividad protectora frente a enfermedades crónicas (diabetes, hipertensión, cataratas, osteoporosis, hipercolesterolemia, disfunción tiroidea y enfermedades renales). El estudio demostró que la vitexina tenía un efecto anti-diabetes (tipo II) y anti-hipercolesterolemia; tilirosida, actividad antidiabética (tipo I), antihipercolesterolemia, antihipóxica, antinefrítica y antiosteoporótica, y Pg-1, anti-catarata, antihipertensiva y anti-tiroidea. La evaluación de la probabilidad del potencial antineoplásico mostró que la vitexina tiene un mejor potencial anticancerígeno en el páncreas, estómago, vejiga y riñón, y tiene un efecto anti-Helicobacter pylori. Pg-1 mostró una mayor actividad anticancerígena en el colon y en la región colon-rectal. La tilirosida, a pesar de mostrar menos efecto que otras sustancias, mostró una actividad anticancerosa pancreática y colon-rectal satisfactoria. Por tanto, estas moléculas pueden convertirse en importantes candidatas a fármacos naturales como recurso terapéutico para combatir enfermedades crónicas y el cáncer de los sistemas gastrointestinal y urinario.

Descriptores: Productos Biológicos; Simulación por Computador; Enfermedad Crónica.

INTRODUÇÃO

Nas últimas décadas houve uma
mudança epidemiológica no padrão de
morbimortalidade da população mundial, com o

aumento da carga de mortalidade por doenças crônicas em comparação às doenças infecciosas ${ }^{1}$. As neoplasias, as doenças 
cardiovasculares e respiratórias crônicas e 0 diabetes mellitus representam o principal grupo de doenças crônicas com elevados índices de mortalidade. Em 2012, 38 milhões de óbitos foram atribuídos a estas doenças sendo que $42 \%$ das mortes registradas foram consideradas prematuras e evitáveis em indivíduos com com menos de 70 anos de idade ${ }^{2}$.

A utilização de práticas não convencionais de saúde, como o uso de plantas medicinais e medicamentos fitoterápicos, pela população adulta para o tratamento de suas enfermidades - é uma situação que vem crescendo em todo o mundo. Além de ser uma opção de baixo custo em relação às drogas alopáticas disponíveis ela encontra-se intrinsecamente atrelada a fortes influências culturais. No Brasil, o uso de plantas e ervas da medicina popular é uma atividade bastante comum, sendo vista como um recurso terapêutico alternativo que envolve conhecimentos herdados sobre o potencial e a especificidade de determinadas espécies vegetais no combate a doenças $e$ microrganismos ${ }^{3}$.

Os fitoterápicos, que são largamente usados na cura de doenças ou no alívio de seus sintomas, têm uma eficácia terapêutica baseada na ação combinada de vários compostos ativos, que buscam reduzir a ocorrência de toxicidade e simultaneamente proporcionar melhorias nas condições de vida, evitando o agravamento de problemas de saúde pré-existentes e efeitos colaterais oriundos de associações medicamentosas. Geralmente, esses efeitos acometem pacientes mais idosos portadores de doenças crônicas, fazendo com que terapias de menor impacto fisiológico sejam mais benéficas em tratamentos com uso prolongado de medicamentos ${ }^{4,5}$.

As substâncias naturais de origem vegetal são fontes promissoras de biomoléculas ativas, como os flavonoides, cuja ação antimicrobiana, antioxidante, anti-inflamatória e imunomoduladora é bem conhecida. Dietas ricas contendo essas substâncias podem auxiliar no combate ao estresse oxidativo, diabetes, inflamação crônica, doenças neurodegenerativas e cardiovasculares ou mesmo câncer ${ }^{6,7}$.

O presente estudo foi desenvolvido com o propósito de avaliar o potencial bioativo in silico dos flavonoides tilirosídeo (Kaempferol-3O- $\beta$-D-(6"-Ep-coumaril) glucopiranosideo), Pg-1 (5,7-dihidroxi-3,8,4'-trimetoxi) e vitexina (5,7,4'-trihidroxiflavona-8-C- $\beta$-glucopiranosideo), abordando a ação preditiva antineoplásica em órgãos do sistema gastrointestinal e urinário, e a atividade protetora contra doenças crônicas (diabetes, hipertensão, catarata, osteoporose, hipercolesterolemia, disfunção de tireoide e doenças renais).

MATERIAL E MÉTODO

- Estrutura química

Os flavonoides analisados foram 0 tlirosideo (Kaempferol-3-O- $\beta$-D-(6"-Ep-coumaril) glucopiranosideo), o Pg-1 (5,7-dihidroxi-3,8,4'trimetoxi) e a vitexina (5,7,4'-trihidroxiflavona-8C- $\beta$-glucopiranosideo). A estrutura química destes compostos foi identificada de acordo com o banco de dados de moléculas Pubchem ${ }^{\circledR}$ (https://pubchem.ncbi.nlm.nih.gov) (Figura 1).

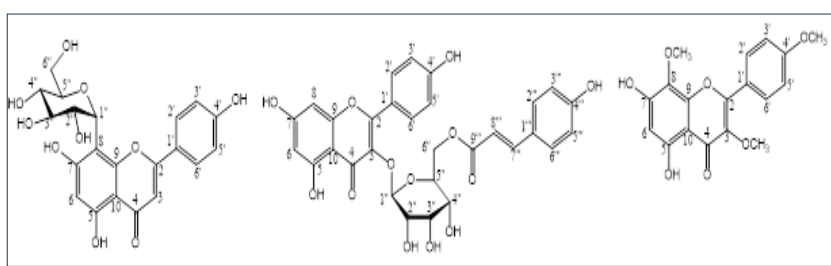

Figura 1. Estrutura química dos flavonoides vitexina, tilirosideo e Pg-1 (Fonte: https://pubchem.ncbi.nlm.nih.gov)

- Análise in silico para previsão dos espectros de atividade das substâncias (PASS)

O software PASS online ${ }^{\circledR}$

(http://www.pharmaexpert.ru/passonline/) foi utilizado para a análise das propriedades bioativas preditivas, que avalia o espectro de atividade de um composto. Esta análise compreende a avaliação da probabilidade de ativação $(\mathrm{Pa})$ ou da probabilidade de inativação (Pi), cujos valores encontram-se entre 0,00 a 1,00. Se a $\mathrm{Pa}$ é maior que $\mathrm{Pi}$, o composto é considerado experimentalmente ativo ${ }^{8}$. Foi abordada a ação preditiva antineoplásica em órgãos do sistema digestivo e urinário mais incidente em pacientes com doenças crônicas. Além disso, foi determinada a atividade protetora contra diabetes, hipertensão, catarata, osteoporose, hipercolesterolemia, disfunção de tireoide e doenças renais.

RESULTADOS E DISCUSSÃO

As análises preditivas das propriedades bioativas da vitexina, tilirosideo e $\mathrm{Pg}-1$ demonstram que as moléculas possuem uma boa probabilidade de ativação $(\mathrm{Pa})$ em relação aos valores de probabilidade de inativação $(\mathrm{Pi})$ para diferentes doenças crônicas, bem como atividade antineoplásica no tratamento do câncer em órgãos do sistema digestivo e excretor.

O presente estudo revelou que o Pg-1 tem melhor probabilidade de ativação para atividade anticatarata, anti-hipertensiva e antitireoidiana; a vitexina apresentou efeito antidiabético sintomático, potencial antidiabético 
(especialmente para o diabetes tipo II) e ação antihipercolesterolemia; 0 tilirosideo obteve melhor índice de atividade antihipercolesterolemia em relação aos demais flavonoides analisados, e mostrou ter propriedade antidiabética (de forma específica para o tipo I), além de outros efeitos, como: antihipóxico, antinefrítico e antiosteoporótico (Tabela 1).

Tabela 1. Probabilidade de fármacos com ação sobre doenças crônicas

\begin{tabular}{|c|c|c|c|c|c|c|}
\hline & \multicolumn{2}{|c|}{ vitexina } & \multicolumn{2}{|c|}{ Tilirosídeo } & \multicolumn{2}{|c|}{ Pg-1 } \\
\hline & $\mathbf{P a}$ & $\mathbf{P i}$ & $\mathbf{P a}$ & $\mathbf{P i}$ & $\mathbf{P a}$ & $\mathbf{P i}$ \\
\hline Anticatarata & --- & --- & 0,138 & 0,117 & 0,157 & 0,103 \\
\hline Antidiabética & 0,783 & 0,005 & 0,581 & 0,014 & 0,195 & 0,170 \\
\hline $\begin{array}{c}\text { Antidiabética (tipo I / } \\
\text { tipo } I^{*} \text { ) }\end{array}$ & $0,173^{*}$ & $0,071^{*}$ & 0,145 & 0,061 & 0,152 & 0,051 \\
\hline $\begin{array}{c}\text { Antidiabética } \\
\text { sintomática }\end{array}$ & 0,508 & 0,007 & 0,395 & 0,014 & 0,352 & 0,020 \\
\hline Antihipercolesterolemia & 0,798 & 0,005 & 0,841 & 0,004 & 0,462 & 0,027 \\
\hline Anti-hipertensiva & 0,173 & 0,112 & 0,163 & 0,142 & 0,196 & 0,063 \\
\hline Antihipóxica & 0,370 & 0,098 & 0,556 & 0,025 & 0,404 & 0,077 \\
\hline Antinefrítica & --- & -- & 0,352 & 0,027 & 0,327 & 0,048 \\
\hline Antiosteoporótica & 0,147 & 0,124 & 0,185 & 0,085 & 0,181 & 0,088 \\
\hline Antitireoidiana & --- & --- & 0,121 & 0,080 & 0,221 & 0,014 \\
\hline
\end{tabular}

Os resultados preditivos da avaliação das atividades farmacológicas do Pg-1 estão em consonância com estudos in vitro e in vivo sobre o potencial farmacológico de flavonoides presentes em extratos de plantas, que indicaram a ação anti-hipertensiva ${ }^{9}$, anticatarata $^{10}$ e antitireoidiana destes compostos $^{11}$. Em relação à vitexina, ensaios in vitro mostraram o efeito antidiabético desse flavonoide, obtido de diferentes tipos de arroz provenientes de um mercado chinês ${ }^{12}$. Além disso, foi também relatado o potencial antidiabético e o efeito anti-hiperlipidêmico da vitexina isolada da espécie Lagenaria siceraria (Molina), em testes in vivo com a utilização de modelos animais de hipertensão e diabetes ${ }^{13}$. Já o tilirosídeo, avaliações in silico realizadas por Luhata \& Luhata $(2017)^{14}$ evidenciaram as propriedades antidiabética e anti-hiperlipidêmica bem como antirreumática dessa substância. As análises in silico deste estudo corroboram os dados sobre o potencial bioativo da vitexina e do tilirosídeo descritos na literatura, além de ressaltar a ocorrência de outros efeitos como o antihipóxico e o antinefrítico.

A análise preditiva do potencial antineoplásico em órgãos dos sistemas urinário e gastrointestinal superior revelou que a vitexina obteve melhor potencial anticâncer na bexiga e na uretra, e no pâncreas e no estômago, respectivamente. Também foi observada a ação sobre a principal bactéria responsável por causar inflamação e lesões gástricas e esofágicas (efeito anti-Helicobacter pylori), que podem aumentar a chance de ocorrência de câncer no sistema gastrointestinal. $\mathrm{O} \mathrm{Pg}-1$ apresentou propriedade anticâncer no cólon e na região colón-retal enquanto o tilirosídeo não demostrou efeito anticâncer significativo nos órgãos analisados, com exceção do câncer colón-retal e pancreático (Tabela 2).

Tabela 2. Probabilidade de fármacos com ação antineoplásica sobre o sistema gastrointestinal e excretor

\begin{tabular}{|ccccccc|}
\hline & \multicolumn{2}{c}{ Vitexina } & \multicolumn{2}{c}{ Tilirosídeo } & \multicolumn{2}{c|}{ Pg-1 } \\
\hline $\begin{array}{c}\text { Anti-Helicobacter } \\
\text { pylori }\end{array}$ & 0,246 & $\mathbf{P i}$ & $\mathbf{P a}$ & $\mathbf{P i}$ & $\mathbf{P a}$ & $\mathbf{P i}$ \\
\hline *câncer gástrico & 0,208 & 0,187 & 0,154 & 0,371 & 0,012 \\
\hline *câncer de pâncreas & 0,330 & 0,040 & 0,327 & 0,042 & 0,268 & 0,087 \\
\hline *câncer de bexiga & 0,220 & 0,029 & 0,176 & 0,077 & 0,213 & 0,034 \\
\hline *câncer renal & 0,224 & 0,028 & 0,220 & 0,030 & 0,167 & 0,078 \\
\hline *câncer de cólon & 0,247 & 0,044 & 0,220 & 0,051 & 0,351 & 0,026 \\
\hline *câncer cólon-retal & 0,260 & 0,045 & 0,236 & 0,051 & 0,372 & 0,025 \\
\hline
\end{tabular}

Os dados apresentados nesta avaliação in silico corroboram os achados em estudos prévios in vitro que destacam 0 efeito antineoplásico no sistema gastrointestinal $\left.\right|^{15}$ e na bexiga $^{16}$ da vitexina. Também respaldam 0 efeito anticâncer na região cólon-retal do tilirosídeo, descrito em ensaios in vitro que avaliaram a atividade de flavonoides contra linhagens de células cancerígenas humanas ${ }^{17}$, e do Pg-1 que possui uma molécula com estrutura análoga que desempenha funções antioxidantes e anticâncer em células de fibrosarcoma ${ }^{18}$. Estes resultados enfatizam as propriedades farmacológicas da vitexina, do tilirosídeo e do Pg-1 para sua possível utilização no tratamento de doenças crônicas e inibição de células cancerígenas, sugerindo um maior alcance da aplicação destes compostos como fármacos promissores em terapias de longa duração.

CONCLUSÃO

A probabilidade de atividade observada na análise in silico deste estudo identificou potencial de ação dos flavonoides avaliados contra doenças crônicas: a vitexina, com potencial antidiabetes (tipo II) e antihipercolesterolemia, o tilirosideo, com atividade antidiabetes (tipo I), antihipercolesterolemia, antihipóxico, antinefrítico e antiosteoporótico, e o Pg-1, com efeito anticatarata, anti-hipertensivo e antitireoidiano. A avaliação da probabilidade do potencial antineoplásico demonstrou que a vitexina possui melhor potencial anticâncer no pâncreas, estômago, bexiga, rim e efeito antiHelicobacter pylori. O Pg-1 apresentou maior potencial anticâncer no cólon e na região colónretal, e o tilirosideo, apesar de exibir menor potencial em relação às demais substâncias, demonstrou atividade satisfatória contra o câncer de pâncreas e cólon-retal. Logo, as moléculas analisadas são candidatas a fármacos com atividade sobre doenças crônicas 
e anticâncer em órgãos do sistema gastrointestinal e urinário.

\section{REFERÊNCIAS}

1. Souza MFM, Malta DC, França EB, Barreto ML. Changes in health and disease in Brazil and its States in the 30 years since the Unified Healthcare System (SUS) was created. Ciênc saúde coletiva. 2018; 23(6):1737-50.

2. Istilli, PT, Teixeira, CRDS, Zanetti, ML, Lima, RAD, Pereira, MCA, Ricci, WZ. Avaliação da mortalidade prematura por doença crônica não transmissível. Rev Bras Enferm. 2020;73(2): e20180440.

3. Lucena, JAS, Guedes, JPM. Uso de fitoterápicos na prevenção e no tratamento da hipertensão arterial sistêmica. REBES. 2020; 10(1):15-22.

4. Sezgin Y. The approach to phytotherapy applications: a case report of hyperkalemia. Acta Endocrinol (Buchar). 2020;16(1):95-96.

5. Li Z, Zhang H, Li Y, Chen H, Wang C, Wong VKW et al. Phytotherapy using blueberry leaf polyphenols to alleviate non-alcoholic fatty liver disease through improving mitochondrial function and oxidative defense. Phytomedicine. 2020;69:153209.

6. Liu YJ, Zhan J, Liu XL, Wang Y, Ji J, He QQ. Dietary flavonoids intake and risk of type 2 diabetes: a meta-analysis of prospective cohort studies. Clin Nutr. 2014;33(1):59-63.

7. Owona BA, Abia WA, Moundipa PF. Natural compounds flavonoids as modulators of inflammasomes in chronic diseases. Int Immunopharmacol. 2020;84:106498.

8. Rakib A, Ahmed S, Islam MA, Haye A, Uddin SMN, Uddin MMN et al. Antipyretic and hepatoprotective potential of Tinospora crispa and investigation of possible lead compounds through in silico approaches. Food Sci Nutr. 2019;8(1):547-56.

9. Montenegro CA, Gonçalves GF, Oliveira Filho AA, Lira AB, Cassiano TTM, Lima NTR et al. In silico study and bioprospection of the antibacterial and antioxidant effects of flavone and its hydroxylated derivatives. Molecules. 2017;22(6):869.

10. Bodakhe SH, Ram A, Verma S, Pandey DP. Anticataract activity of rhamnocitrin isolated from Bauhinia variegata stem bark. Orient Pharm Exp Med. 2012;12(3):227-32.

11. Ferreira AC, Rosenthal D, Carvalho DP. Thyroid peroxidase inhibition by Kalanchoe brasiliensis aqueous extract. Food Chem Toxicol. 2000;38(5):417-21.

12. Yao $Y$, Cheng $X Z$, Wang $L X$, Wang $S H$, Ren $G$. Major phenolic compounds, antioxidant capacity and antidiabetic potential of rice bean (Vigna umbellata L.) in China. Int $\mathrm{J}$ Mol Sci. 2012;13(3):2707-16 .
13. Mali VR, Mohan V, Bodhankar SL. Antihypertensive and cardioprotective effects of the Lagenaria siceraria fruit in NG-nitro-Larginine methyl ester (L-NAME) induced hypertensive rats. Pharm Biol. 2012;50(11):1428-35.

14. Luhata, LP, Luhata, WG. Tiliroside: biosynthesis, bioactivity and structure activity relationship (SAR)-A review. J Phytophatmacol, 2017;6:343-8.

15. Xue HF, Ying ZM, Zhang WJ, Meng YH, Ying $X X$, Kang TG. Hepatic, gastric, and intestinal first-pass effects of vitexin in rats. Pharm Biol. 2014;52(8):967-71.

16. Scarpa ES, Emanuelli M, Frati A, Pozzi V, Antonini E, Diamantini G, Di Ruscio G, Sartini D, Armeni T, Palma F, Ninfali P. Betacyanins enhance vitexin-2-O-xyloside mediated inhibition of proliferation of T24 bladder cancer cells. Food Funct. 2016;7(12):4772-80.

17. Tsimplouli C, Demetzos C, HadzopoulouCladaras $M$, Pantazis $\mathrm{P}$, Dimas K. In vitro activity of dietary flavonol congeners against human cancer cell lines. Eur $J$ Nutr. 2012;51(2):181-90.

18. Jahaniani F, Ebrahimi SA, Rahbar-Roshandel $\mathrm{N}$, Mahmoudian M. Xanthomicrol is the main cytotoxic component of Dracocephalum kotschyii and a potential anti-cancer agent. Phytochemistry. 2005;66(13):1581-92.

\section{CONFLITO DE INTERESSES}

Os autores declaram não haver conflitos de interesse

AUTOR PARA CORRESPONDÊNCIA

Aleson Pereira de Sousa
ORCID: https://orcid.org/0000-0002-3430-477X
Universidade Federal da Paraíba (UFPB), Brasil
E-mail: aleson_155@hotmail.com

Submetido em 03/09/2020 Aceito em 02/03/2021 